Article

\title{
Enteric Hard Capsules for Targeting the Small Intestine: Positive Correlation between In Vitro Disintegration and Dissolution Times
}

\author{
Maoqi Fu ${ }^{\dagger}$, Jozef Al-Gousous ${ }^{\dagger}$, Johannes Andreas Blechar and Peter Langguth * \\ Department of Biopharmaceutics and Pharmaceutical Technology, Johannes Gutenberg University Mainz, \\ D-55099 Mainz, Germany; maoqifu1@uni-mainz.de (M.F.); joalgous@uni-mainz.de (J.A.-G.); \\ jblechar@uni-mainz.de (J.A.B.) \\ * Correspondence: langguth@uni-mainz.de \\ t These authors contributed equally.
}

Received: 10 December 2019; Accepted: 31 January 2020; Published: 3 February 2020

\begin{abstract}
In this study, the potential for correlation between disintegration and dissolution performance of enteric-coated (EC) dosage forms was investigated. Different enteric hard shell capsule formulations containing caffeine as model drug were tested for disintegration (in a compendial disintegration tester) and for dissolution in both USP type I (basket) and type II (paddle) apparatuses using different media. Overall, good correlations were obtained. This was observed for both the basket and the paddle apparatus, indicating that the use of disintegration testing as a surrogate for dissolution testing (allowed by International Conference on Harmonization (ICH) for immediate release dosage forms in case, in addition to other conditions, a correlation between disintegration and dissolution is proven) could be extended to include delayed release dosage forms.
\end{abstract}

Keywords: disintegration; dissolution; enteric-coated; ICH; quality control

\section{Introduction}

Disintegration tests have been used to evaluate dosage form performance since the early 20th century, with the current compendial disintegration tester being available since the 1950s [1]. Despite the limitation of the released amount of the active pharmaceutical ingredient (API) not being measured, these tests are still widely used in pharmaceutical practice owing to their simplicity and speediness compared to dissolution tests. This contributed to the International Conference on Harmonization (ICH) allowing disintegration tests to be used as dissolution test surrogates if (among other conditions) a correlation between disintegration and dissolution is proven [2].

In this regard, it appears from the literature data that the likelihood of obtaining a disintegration-dissolution correlation varies greatly from formulation to formulation, with Gupta et al. [3] testing a 12 different verapamil hydrochloride formulations and finding that only one of them gave a satisfactory correlation. Radwan et al. [4] investigated different trospium chloride formulations under different conditions and found that a correlation is possible when the disintegration is not too rapid. Nickerson et al. [5] on the other hand found good correlations for several immediate formulations of an unnamed API. However, the focus has generally been restricted to immediate release dosage forms, most probably because of the ICH guidance restricting the possibility of employing disintegration test as a dissolution test surrogate to non-modified release dosage forms.

However, one class of modified release dosage forms, namely enteric-coated (EC) formulations, offers at least a theoretical possibility for obtaining good disintegration dissolution correlations. For, in the presence of a rapidly disintegrating and dissolving core, having a situation where the disintegration 
of the enteric coat strongly influences the overall release performance is likely. Therefore, this work is going to investigate the correlation between disintegration and dissolution of enteric-coated hard shell capsules in order to explore the feasibility of employing the disintegration test as a dissolution test surrogate for EC dosage forms.

\section{Materials and Methods}

\subsection{Materials}

Hydroxypropyl methylcellulose size 0 capsules (ACG Nature Caps Plus) were received form ACG Associated Capsules Pvt Ltd (Mumbai, India). DRcaps ${ }^{\circledR}$ (nutraceutical capsules with inherent enteric properties of the capsule shell) were obtained from Neue Lebensqualität (Badendorf, Germany). Caffeine (median particle size $48 \mu \mathrm{m}$ ) and magnesium stearate were purchased from Caesar \& Loretz GmbH (Hilden, Germany). Fumed silica was purchased from Fagron GmbH \& CO.KG (Glinde, Germany). Hypromellose phthalate (HP-50), hypromellose acetate succinate (HPMCAS-HG, AQOAT) and low substituted hydroxypropyl cellulose (L-HPC) were received as a gift from Shin-Etsu (Wiesbaden, Germany). Lactose (FlowLac ${ }^{\circledR}$ 90) was received from Molkerei Meggle (Wasserburg, Germany). Triethyl citrate (TEC) was purchased from Sigma-Aldrich (Overijse, Belgium). Talc was purchased from Imerys (Luzenac, France). All other materials were of analytical grade.

\subsection{Capsule Filling}

The HPMC capsules and DRcaps ${ }^{\circledR}$ were filled manually (aponorm ${ }^{\circledR}$ Kapselfüllgerät, WEPA Apothekenbedarf GmbH \& CO.KG, Hillscheid, Germany) with a powder formulation. The powder formulation was prepared using a 1.6 L Turbula mixer (Willy A. Bachofen GmbH, Muttenz, Switzerland) at $49 \mathrm{rpm}$ (batch size $600 \mathrm{~g}$ ), and its composition is described in Table 1. The capsules were filled with $375 \mathrm{mg}$ of the powder (i.e., $75 \mathrm{mg}$ of caffeine). The filled capsules complied with the content uniformity requirements of the European Pharmacopoeia 9.0.

Table 1. Formulation of the capsule filling. All values (\%) are based on the total weight $(\mathrm{m} / \mathrm{m})$.

\begin{tabular}{ccc}
\hline Substance & $\mathbf{\%}(\mathbf{m} / \mathbf{m})$ & Weights of the Components per Capsule $(\mathbf{m g})$ \\
\hline Caffeine & 20 & 75 \\
\hline L-HPC & 15 & 56.25 \\
\hline Lactose & 63.75 & 239.06 \\
\hline Silica & 0.25 & 0.94 \\
\hline Magnesium stearate & 1 & 3.75 \\
\hline Total & 100 & 375 \\
\hline
\end{tabular}

\subsection{Capsule Coating}

The ACG Nature Caps Plus were coated. Two different batches of coated capsules were prepared from them. The coating formulations are described in Table 2. First, the ethanol-water solution was prepared. The polymer was dissolved in $80 \%$ of the solvent and the remaining $20 \%$ of the solvent were used to disperse the talc. Afterwards, the polymer solution is combined with the talc dispersion. Last, triethyl citrate is added to the formulation (in case of the HPMCAS-HG formulation). Before coating, the polymer solution is filtered using a sieve with a pore size of $0.2-0.4 \mathrm{~mm}$. The coating levels of the capsules coated with HP-50 and HPMCAS-HG are $10 \mathrm{mg}$ polymer/cm ${ }^{2}$ and $9 \mathrm{mg}$ polymer/cm $\mathrm{cm}^{2}(30 \%$ and 27\% weight gain), respectively. A Solidlab 1 drum coater (Robert Bosch Packaging Technology GmbH, Waiblingen, Germany) was used for coating with the following parameters: $230 \mathrm{~g}$ of capsules/batch preheated to $30{ }^{\circ} \mathrm{C}$; spray rate of $6.5-7 \mathrm{~g} / \mathrm{min}$ and a atomizing pressure of 2.0 bar; nozzle diameter of 
$0.5 \mathrm{~mm}$; the inlet air was heated to $58-60{ }^{\circ} \mathrm{C}$ and had a flow rate of $55 \mathrm{~m}^{3} / \mathrm{h}$; product temperature of $35-38{ }^{\circ} \mathrm{C}$.

Table 2. Coating formulation. All values (\%) are based on the total solution.

\begin{tabular}{ccccc}
\hline \multirow{2}{*}{ Substance } & \multicolumn{3}{c}{ HP-50 Formulation } & \multicolumn{2}{c}{ HPMCAS-HG Formulation } \\
\cline { 2 - 5 } & $\mathbf{\%} \mathbf{( m / m )}$ & Weights of the Components $\mathbf{( g )}$ & $\mathbf{\%}(\mathbf{m} / \mathbf{m})$ & Weights of the Components $(\mathbf{g})$ \\
\hline Polymer & 6 & 60 & 5 & 40 \\
\hline Talc & 7.5 & 75 & 7.5 & 60 \\
\hline TEC & - & - & 2 & 16 \\
\hline Ethanol & 69.2 & 747.7 & 68.4 & 591.25 \\
\hline Water & 17.3 & 117.3 & 17.1 & 92.75 \\
\hline
\end{tabular}

\subsection{Disintegration Test}

The capsule disintegration was performed with disks using a DT2 Disintegration Tester (Sotax AG, Aesh, Switzerland) complying with the European Pharmacopoeia specifications for a type A disintegrat ion testing apparatus. All capsules were first exposed to $700 \mathrm{~mL}$ of $0.1 \mathrm{M} \mathrm{HCl}$ for one hour followed by one hour testing in $700 \mathrm{~mL}$ of buffer. The temperature was maintained at $37.0 \pm 0.5^{\circ} \mathrm{C}$ There were three different buffers tested, namely the $50 \mathrm{mM}$ USP phosphate buffer pH 6.8, blank FaSSIF buffer (28.4 mM) pH 6.5 and a $15 \mathrm{mM}$ phosphate buffer $\mathrm{pH} 6.5$ that showed to be biopredictive in previous studies (henceforth referred to as the "Al-Gousous et al. medium" [6]). The disintegration times recorded are the times at which the capsules ruptured, which helps reduce the uncertainty associated with determining the disintegration times based on "complete disintegration" [7]. Accordingly, these times are defined as the times at which first visible cracks in the capsule shell appear. In order to avoid observer bias, disintegration tests were performed before dissolution tests.

\subsection{Acid Uptake Test}

Six capsules were individually weighed and then tested in a disintegration tester as outlined above (the Disintegration Test subsection) but without disks, and only in $\mathrm{HCl}(0.1$ and $0.01 \mathrm{M})$ for one and two hours. Sinkers (Japanese Pharmacopoeia Standard, Pharma Test, Hainburg, Germany) were used to prevent the capsules from floating. At the end of the test the capsules were removed, blotted and the \%weight gain was calculated as follows:

$$
\% \text { weight gain in acid }=\frac{\text { mass after acid exposure }- \text { mass before acid exposure }}{\text { mass before acid exposure }} \times 100 \%
$$

\subsection{Dissolution Test}

The drug release was tested with a DT6R dissolution tester (Erweka GmbH \& CO.KG, Langen, Germany). The device was used as a USP type I dissolution tester at $100 \mathrm{rpm}$ as well as a USP type II dissolution tester with sinkers (same as in previous subsection) at $50 \mathrm{rpm}$. The use of sinkers prevented the capsules from floating in the paddle apparatus. In accordance with the disintegration test, the capsules were studied for one hour in $0.1 \mathrm{M} \mathrm{HCl}$ followed by a media change to either one of the buffers described previously. The volume of the dissolution media was $700 \mathrm{~mL}$. The temperature was maintained at $37.0 \pm 0.2^{\circ} \mathrm{C}$. The $5 \mathrm{~mL}$ samples were filtered through a $0.8 \mu \mathrm{m}$ cellulose acetate nitrate filter (Rotilab Spritzenfilter CME, Carl Roth, Karlsruhe, Germany). The first $1 \mathrm{~mL}$ of the filtrate was discarded to saturate the membrane. Blank buffer was used to replace the sample volume. Caffeine was quantified spectrophotometrically at $\lambda=275 \mathrm{~nm}$. 


\subsection{Correlation between Disintegration and Dissolution Results}

Disintegration times were correlated with the times required to achieve $10 \%, 50 \%$ and $80 \%$ release ( $\mathrm{t} 10 \%, \mathrm{t} 50 \%$ and $\mathrm{t} 80 \%$ respectively) representing the early, middle and late portions of the dissolution profiles. The aforementioned times were calculated using linear interpolation. The correlation was done using simple linear regression performed by Microsoft Excel (Microsoft Office 2013, Microsoft Corporation, Redmond, WA, USA). Hypothesis testing was performed on the slope using a one-sided $t$-test (With the null hypothesis being slope $=0$ ). One-sided $p$-values were calculated since a positive correlation is expected. The hypothesis testing was performed using the vassarstats website [8].

\section{Results and Discussion}

\subsection{Disintegration and Acid Uptake}

As shown in Figure 1, DRcaps@Enteric gave the fastest disintegration while HPMCAS-HG gave the slowest. Disintegration tended to be fastest in the USP dissolution testing medium and slowest in the Al-Gousous et al. medium as would be expected based on the buffer molarities of the media. The fast disintegration of DRcapsßEnteric, however, seems to be associated with poor resistance to acid as evidenced by the acid uptake values shown in Table 3 and by the deformation exhibited by those capsules (Figure 2). This indicates that it is rather the weakened capsule shell structure that results in rapid disintegration in buffer. This is rather in line with the findings of Al-Tabakha et al. [7], where even rupture of such capsule shells was observed in simulated gastric fluid.

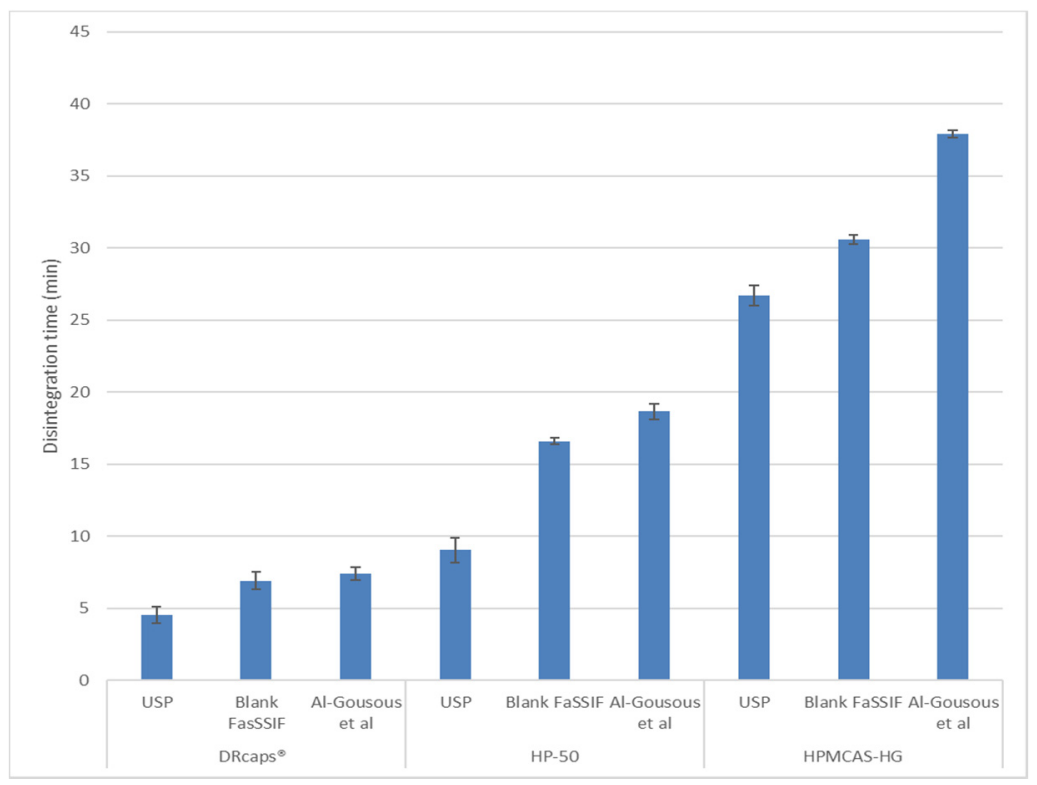

Figure 1. Disintegration times (mean $\pm S D)$ of the tested formulations $(n=6)$.

Table 3. Weight gain (mean \pm SD) of the tested formulations $(n=6)$ after $1 \mathrm{~h}$ in acidic media.

\begin{tabular}{|c|c|c|c|c|}
\hline \multirow{2}{*}{ Formulation } & \multicolumn{2}{|c|}{$\%$ Weight gain in $0.1 \mathrm{M} \mathrm{HCl}$} & \multicolumn{2}{|c|}{$\%$ Weight gain in $0.01 \mathrm{M} \mathrm{HCl}$} \\
\hline & After $1 \mathrm{~h}$ & After $2 \mathrm{~h}$ & After $1 \mathrm{~h}$ & After $2 \mathrm{~h}$ \\
\hline DRcaps $®$ & $6.5 \pm 0.7$ & $7.4 \pm 0.3$ & $11.2 \pm 0.2$ & $13.5 \pm 0.6$ \\
\hline HP-50 & $3.4 \pm 0.6$ & $4.2 \pm 0.3$ & $3.8 \pm 0.1$ & $6.1 \pm 0.7$ \\
\hline HPMCAS-HG & $2.5 \pm 0.4$ & Ruptured & $2.8 \pm 0.6$ & Ruptured \\
\hline
\end{tabular}




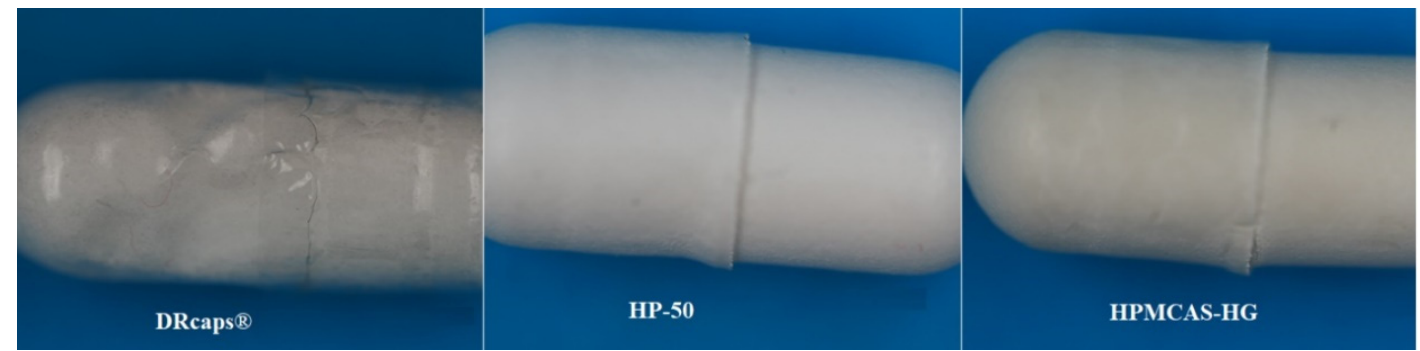

Figure 2. Appearance of DRcaps®and coated capsules after $1 \mathrm{~h}$ in $0.1 \mathrm{M} \mathrm{HCl}$.

It is interesting that despite showing the lowest acid uptake at one hour, the HPMCAS-HG capsules ruptured in acid within roughly $1.5-2 \mathrm{~h}$ during the acid uptake tests. This might be related to mechanical instability. Figure 2 shows that while the DRCaps®show extensive deformation, HP-50-coated ones show only some wear at the gap between the body and the cap. HPMCAS-HG-coated capsules behave in a manner similar to the HP-50-coated ones but the wear at the gap seems to be a bit more extensive, which may impart mechanical instability to the capsule. The causes behind this need to be further investigated.

As shown in Figure 1, after $1 \mathrm{~h}$ in acid, the capsules coated with HPMCAS-HG still show the longest disintegration times. Only the high buffer capacity USP medium [6] showed large acceleration in disintegration compared to the situation where testing in acid was continued for one further hour (in the acid uptake tests with sinkers and without disks). This further supports the mechanical instability hypothesis. As for why the presence of disks in the disintegration test (compared to their absence in the acid uptake test) does not seem to have a dramatic effect, this might be related to the disk impacting the capsule from above rather than tearing it apart. Other factors could be the force generated on contact between the capsules and the sinkers in the disk-free setup as well as the tilted orientation of the capsules in the disintegration tester tubes when inside sinkers and its potential effects on hydrodynamics. Further investigation is needed regarding this issue, which is outside the scope of this manuscript.

\subsection{Dissolution}

As shown in Figure 3, the dissolution results followed the trends exhibited by the disintegration times. The disintegration times are lesser even than the $10 \%$ values, which is most probably associated with the capsule rupture being a pre-requisite for significant drug release and with the greater mechanical stresses in the disintegration tester [9]. Dissolution tended to be slower in the basket apparatus. This may be associated with the lower fluid velocities in the central and upper regions of the basket (at $100 \mathrm{rpm}$ ) where the capsule tends to be located owing to its buoyancy compared to the bottom of the vessel in a paddle apparatus (at $50 \mathrm{rpm}$ ) [10]. This implies that hydrodynamic differences between the different apparatuses play an important role in the obtained correlations. 


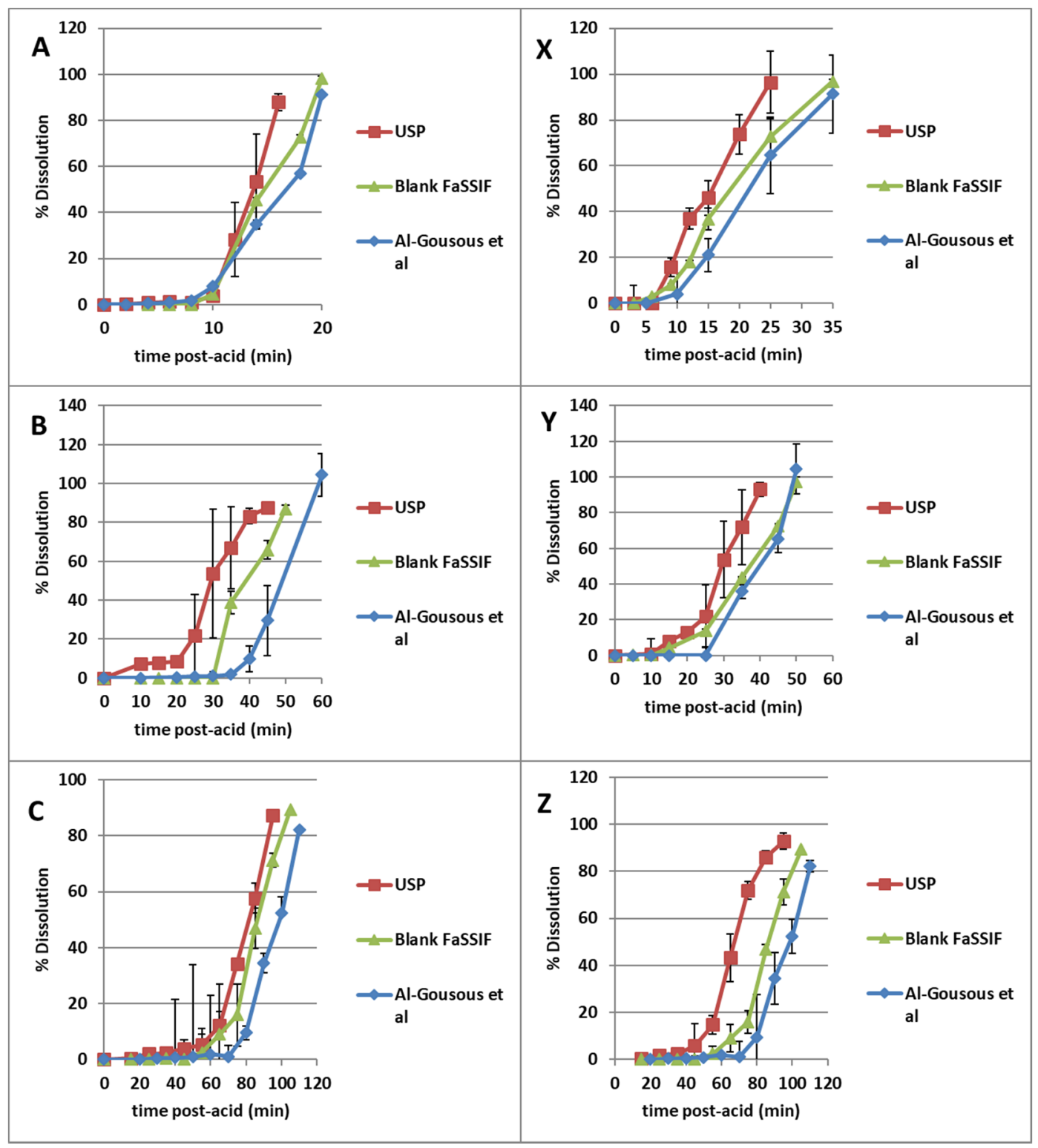

Figure 3. Dissolution test results (mean $\pm S D)$ of the tested formulations $(n=6)$. Panels $(A-C)$ represent DRcaps $囚, H P-50$ and HPMCAS-H respectively in basket apparatus, while panels $\mathbf{X}, \mathbf{Y}$ and $\mathbf{Z}$ represent DRcaps®, HP-50 and HPMCAS-H respectively in paddle apparatus.

\subsection{Correlation between Disintegration and Dissolution Results}

Disintegration times were correlated with the times required to achieve 10, 50 and $80 \%$ release ( $\mathrm{t} 10 \%, \mathrm{t} 50 \%$ and $\mathrm{t} 80 \%$ respectively) representing the early, middle and late portions of the dissolution profiles. When all the dissolution times were correlated with their respective disintegration times, good overall correlations were obtained for all dissolution profile portions (Figure 4). 

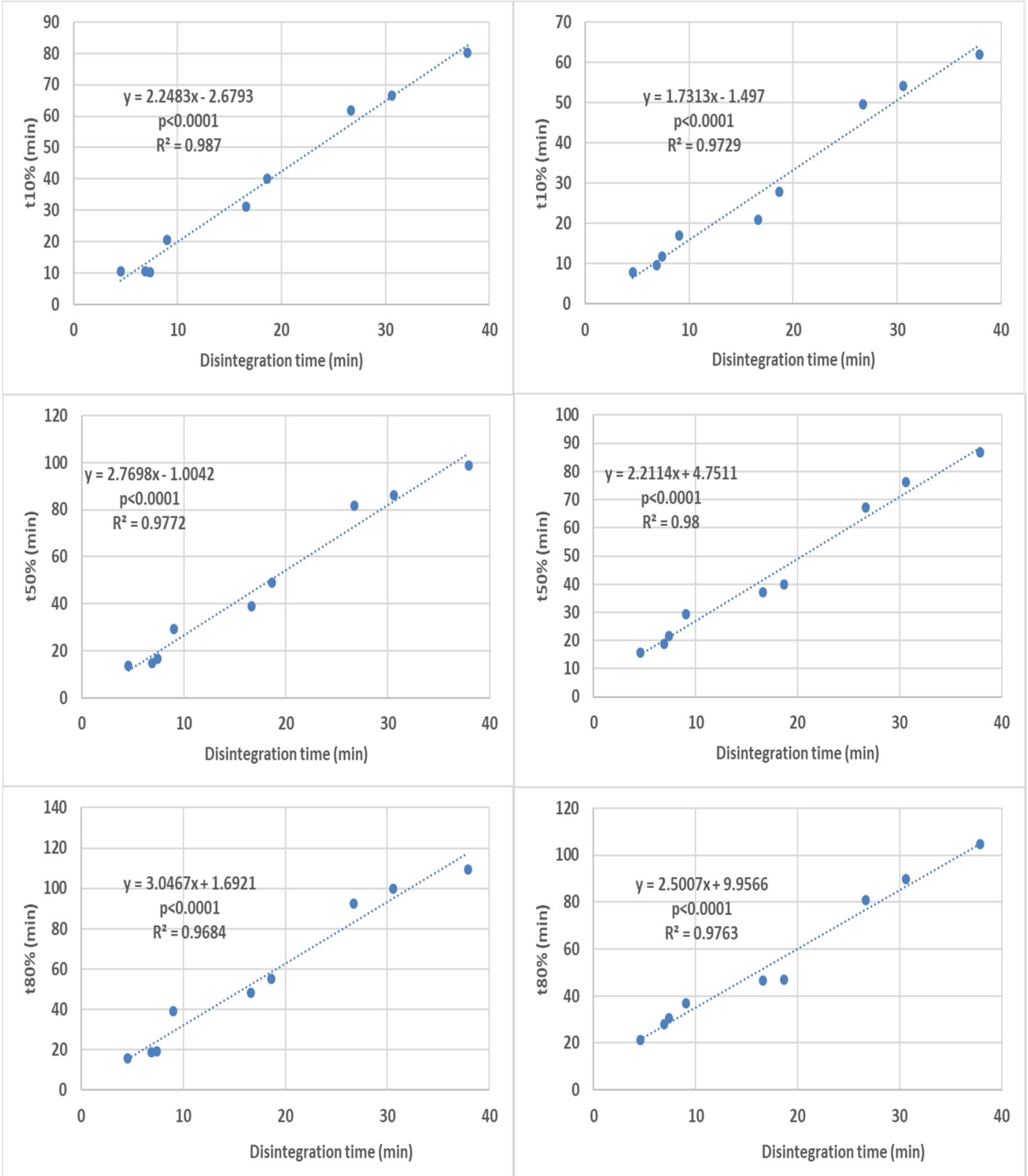

Figure 4. Overall correlation of all the disintegration results with their respective dissolution results (all formulations in all media are present in each graph) in the basket apparatus (right-hand side) and the paddle apparatus (left-hand-side). The $p$-value is a one-sided value for a $t$-test applied to the slope.

A more detailed analysis was performed by making three point correlations for formulation effects (Figures 5 and 6) and medium effects (Figures 7 and 8). When the results of different formulations tested in the same medium were correlated, good $\mathrm{r}^{2}$ and $\mathrm{p}$-values were almost invariably obtained. However, the situation was different when correlating results of the same formulation in different media (Figures 8 and 9), where the differences tended to be smaller than the inter-formulation differences.

Poor correlations were generally obtained for DRcaps®(with the notable exception of the $t 80 \%$ case). A possible explanation for that could be that the weakened capsule shell structure made the initial rupture more associated with random mechanical events and less with the enteric-polymer dissolution promoting capabilities of the buffer. The complete process of shell dissolution/disintegration was less confounded by such random effects resulting in better correlations for $t 80 \%$. As for the weak correlations obtained for the $\mathrm{t} 10 \%$ and $\mathrm{t} 80 \%$ parameters for the HP-50-coated capsules, they seem to be 
caused by the close disintegration times in the blank FaSSIF and Al-Gousous et al. media. This is most probably related to the different discriminative abilities of the disintegration tester vs. paddle and basket apparatuses (owing to the different hydrodynamics). HPMCAS-HG gave the best correlations likely due to the slow capsule shell disintegration relative to drug dissolution. Anyway, despite multiple instances of weak correlations, when correlating the results of one formulation in different media, each formulation shows at least one instance with $p<0.05$.

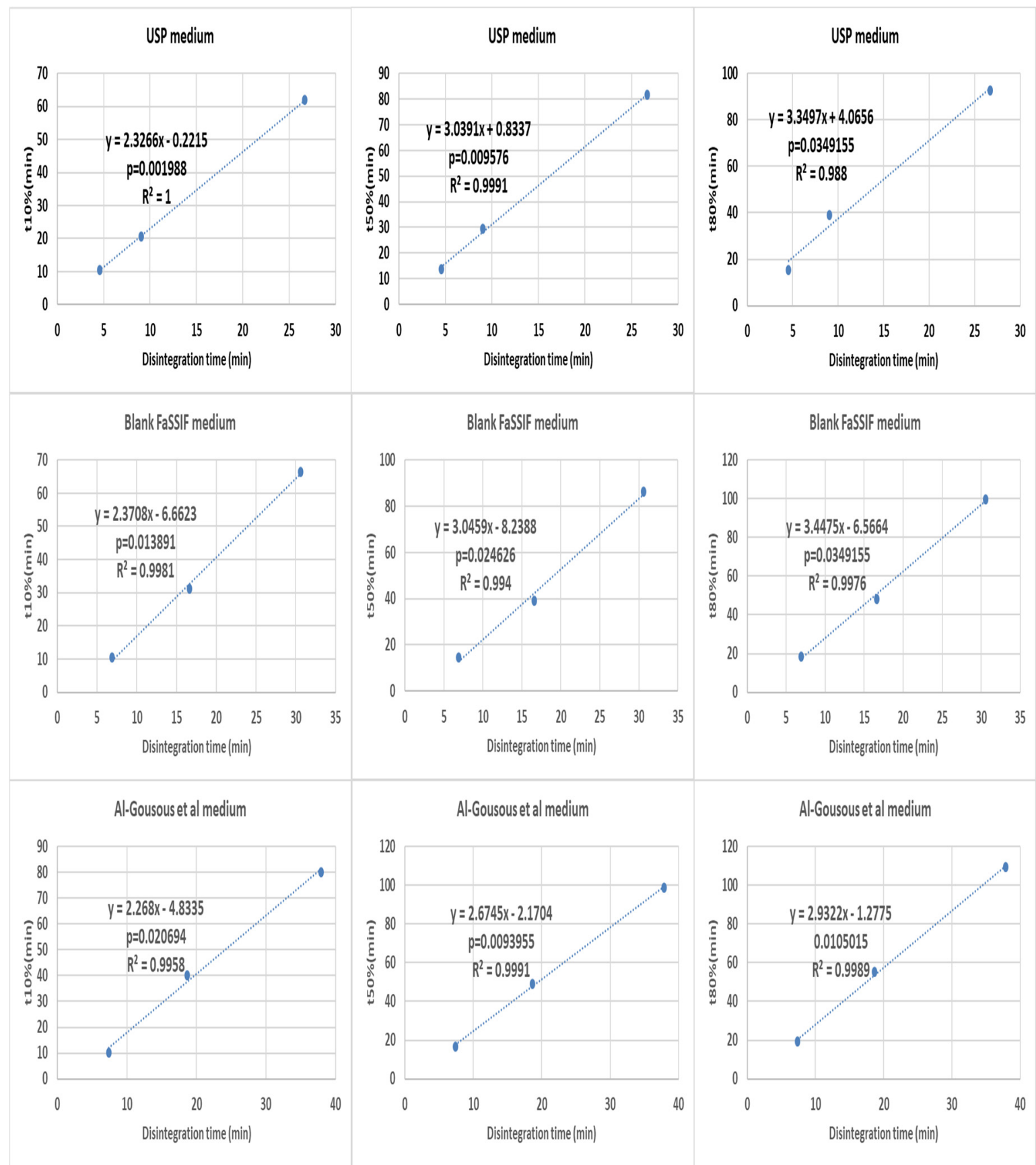

Figure 5. Correlating disintegration times with different dissolution parameters (basket apparatus) for different formulations tested in one medium. The $p$-value is a one-sided value for a $t$-test applied to the slope. 


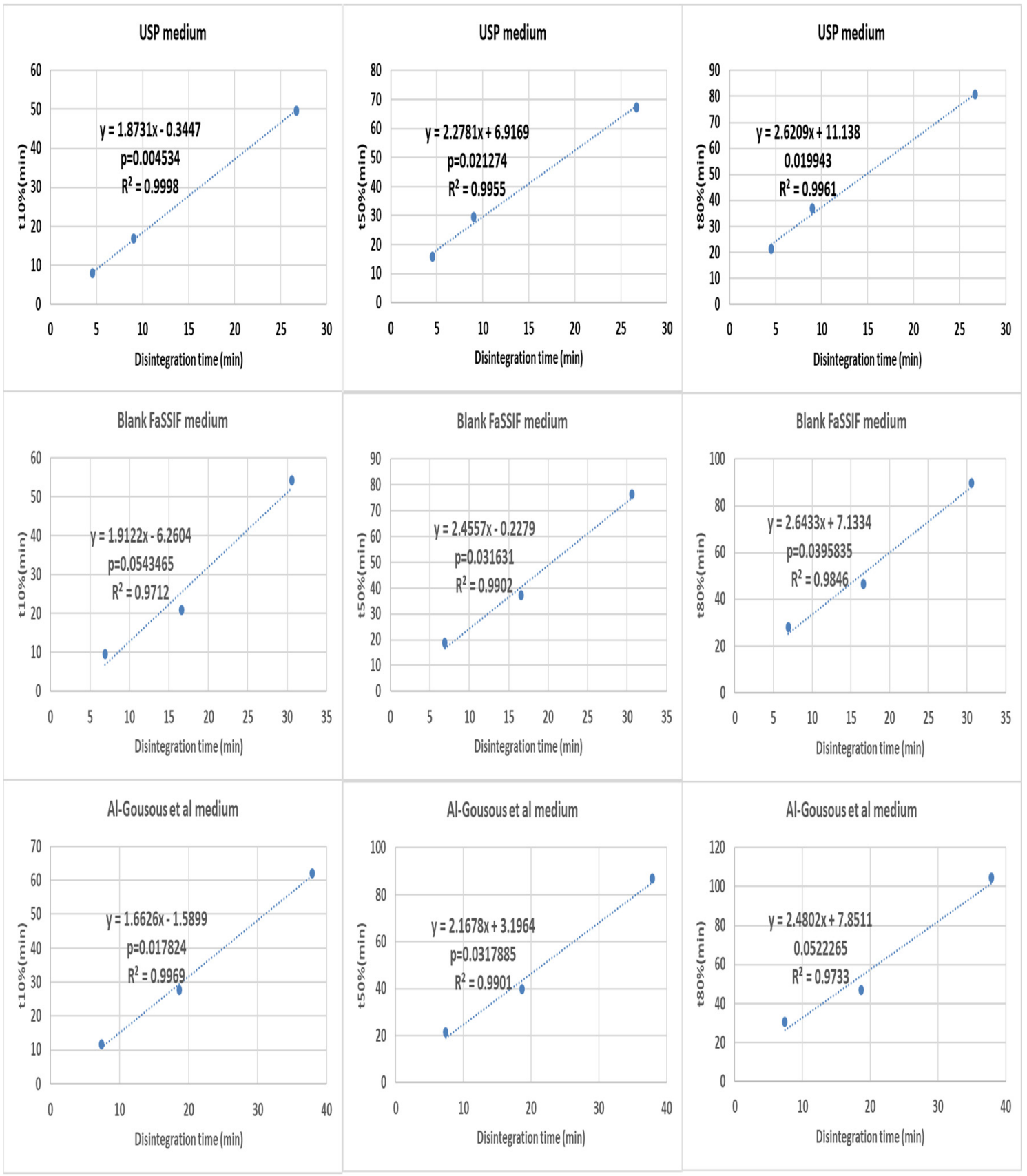

Figure 6. Correlating disintegration times with different dissolution (paddle apparatus) parameters for different formulations tested in one medium. The $p$-value is a one-sided value for a $t$-test applied to the slope. 


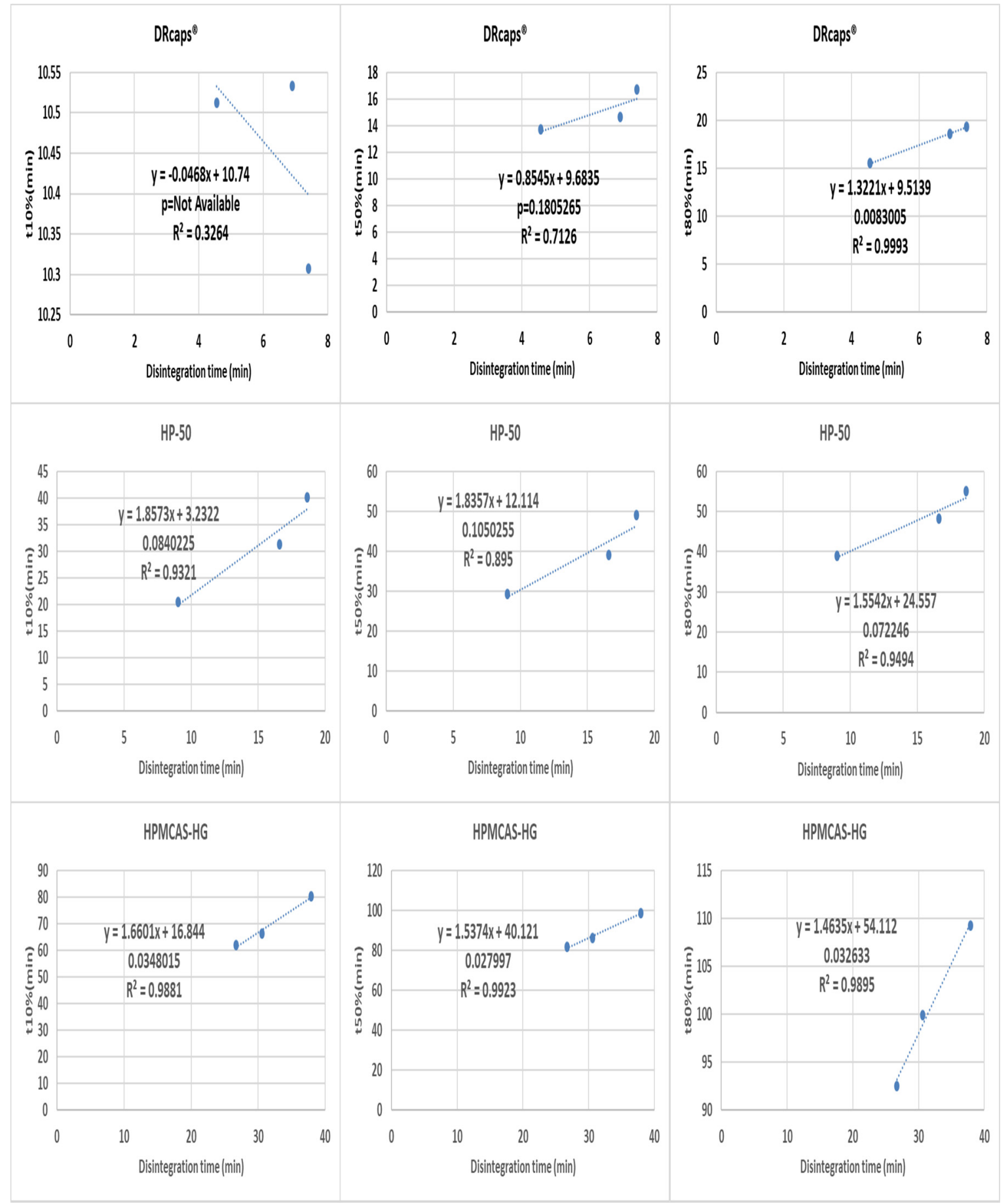

Figure 7. Correlating disintegration times with different dissolution (basket apparatus) parameters for one formulation in different media. The $p$-value is a one-sided value for a $t$-test applied to the slope. 


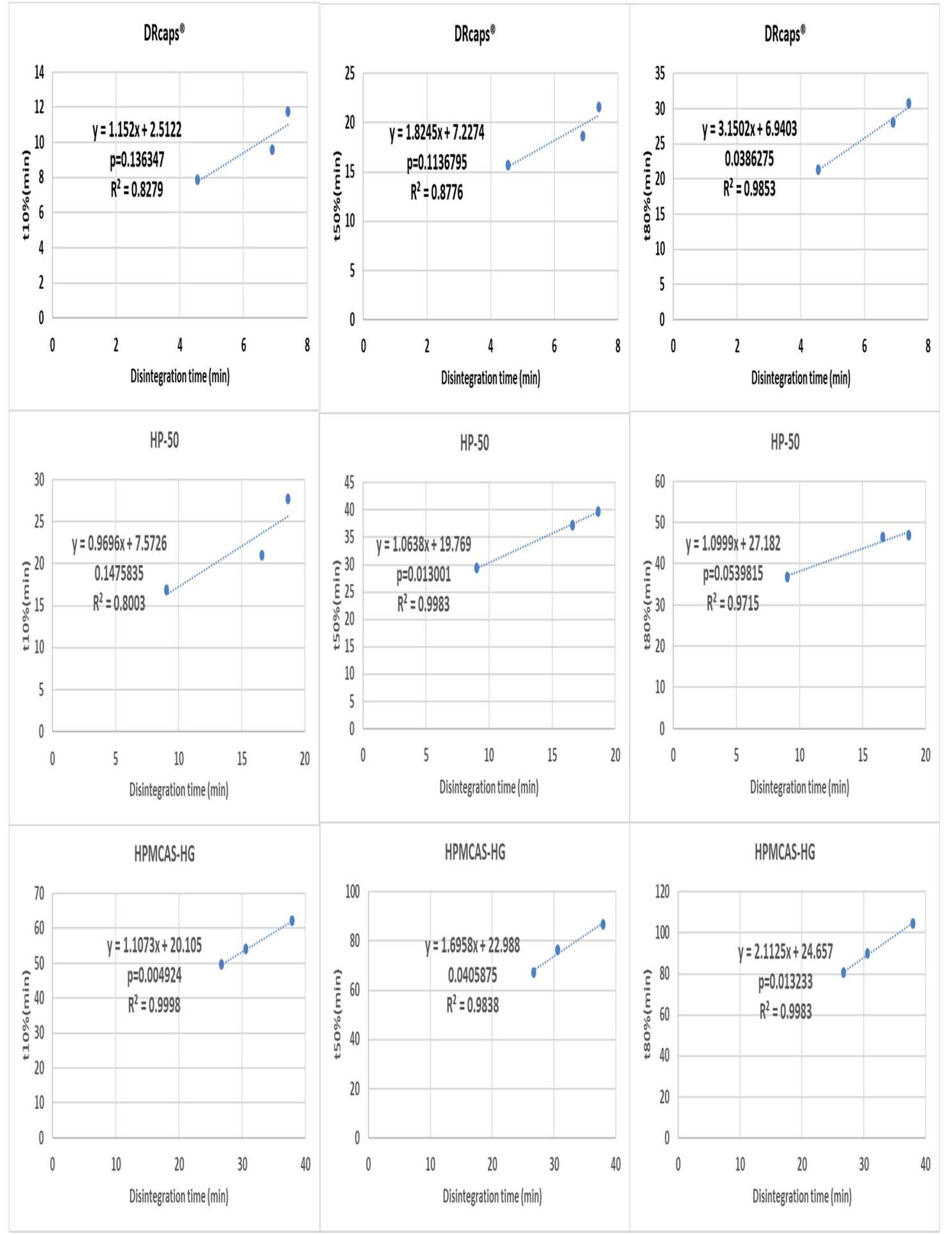

Figure 8. Correlation of disintegration times with different dissolution (paddle apparatus) parameters for one formulation in different media. The $p$-value is a one-sided value for a $t$-test applied to the slope. 

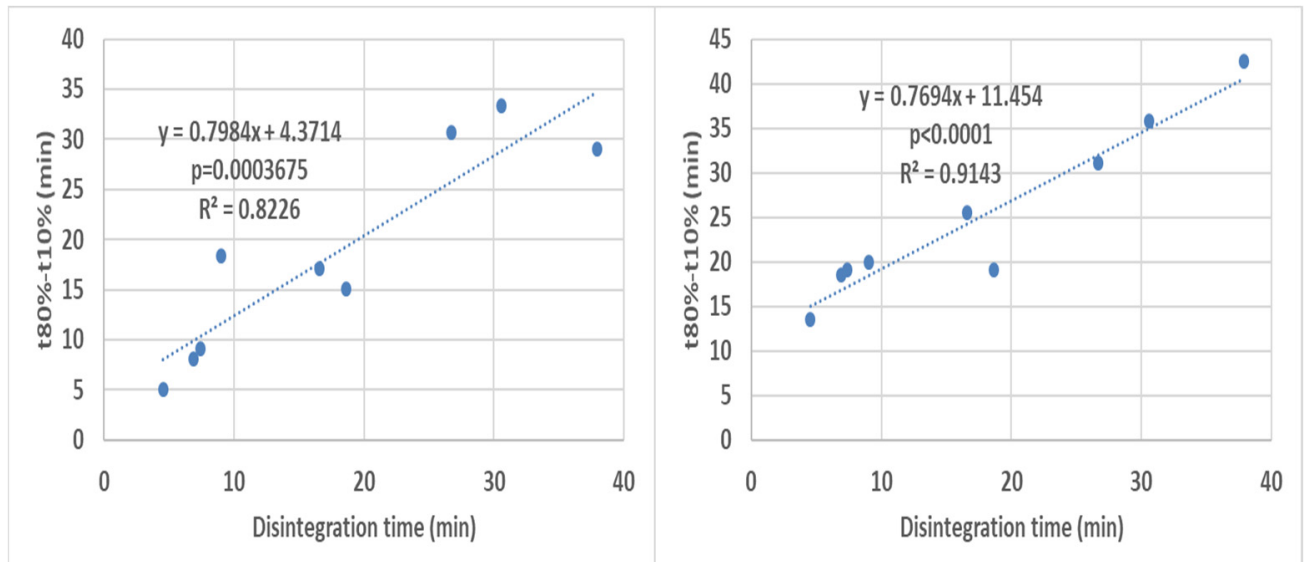

Figure 9. Overall correlation of all the disintegration times with their ( $\mathrm{t} 80 \%-\mathrm{t} 10 \%)$ values results (all formulations in all media are present in each graph) in the basket apparatus (right-hand side) and the paddle apparatus (left-hand-side). The $p$-value is a one-sided value for a $t$-test applied to the slope.

An additional observation is that the correlations for different dissolution time points show not only different intercepts but also different slopes. This indicates that the disintegration times do not correlate with the different dissolution times solely because of the profiles being shifted because of different coat rupture times, but also because of the influence of the disintegration on the overall post-capsule rupture release kinetics. This is shown by the fair to strong overall correlations obtained for the difference between $\mathrm{t} 80 \%$ and $\mathrm{t} 10 \%$ (corresponding to the time required for $\%$ release to rise from $10 \%$ to $80 \%$ ).

Figure 9 shows stronger correlation for the paddle apparatus. A possible reason could be that variation in the floating capsule orientation inside the basket, together with the more variable fluid velocities in the upper region of the basket [10], leads to the greater data point scatter observed for the basket apparatus.

All in all, the obtained set of correlations shows that enteric-coated formulations seem promising with regard to using disintegration tests as dissolution surrogates. This shows that the use of disintegration testing as dissolution testing surrogate might not have to be restricted only to immediate release dosage forms. However, further investigations on further EC dosage forms need to be performed before making a definitive judgment on this matter.

\section{Conclusions}

Obtaining good correlations between the dissolution and disintegration results of EC dosage forms is possible. This opens the way for more rigorous research that could help in expanding the dissolution test waiver concept beyond immediate release dosage forms. Further investigations on additional EC formulations could help to establish regulatory criteria regarding this matter. However, extrapolating these findings to the in vivo situation should be done with extreme caution since many factors like (among others) possible transporter saturation effects [11], interplay with food and gastric emptying effects [12,13] as well as different hydrodynamics and mechanical stresses [9] complicate the correlation between disintegration and bioavailability. These issues need to be taken into account when considering an expanded role for disintegration testing in product evaluation.

Author Contributions: Data curation, J.A.-G.; Formal analysis, P.L.; Investigation, M.F. and J.A.B.; Project administration, P.L.; Resources, P.L.; Supervision, P.L.; Writing—original draft, J.A.-G. and P.L. All authors have read and agreed to the published version of the manuscript.

Funding: This research received no external funding.

Acknowledgments: This work was supported by a scholarship from China government (China Scholarship Council, CSC). We are grateful to Shin-Etsu Tylose GmbH \& Co. (Wiesbaden, Germany) for the use of drum 
coater and the scanning electron microscope and supplying Aqoat $®$ AS-LG, MG, HG, HPMCP coating polymers. Special appreciation is expressed to Andreas Sauer for the advice in the whole processing and to Ilja. Lesser and Tobias Eggers for their technical assistance and support. We would also like to thank ACG Associated Capsules (Mumbai, India) for supplying the ACG Nature Caps Plus.

Conflicts of Interest: The authors declare no conflict of interest.

\section{References}

1. Al-Gousous, J.; Langguth, P. Oral Solid Dosage Form Disintegration Testing-The Forgotten Test. J. Pharm. Sci. 2015, 104, 2664-2675. [CrossRef] [PubMed]

2. International Conference on Harmonization. Available online: https://database.ich.org/sites/default/files/ Q6A_Guideline.pdf (accessed on 28 November 2019).

3. Gupta, A.; Hunt, R.L.; Shah, R.B.; Sayeed, V.A.; Khan, M.A. Disintegration of highly soluble immediate release tablets: A surrogate for dissolution. Aaps Pharm. Sci. Tech. 2009, 10, 495-499. [CrossRef] [PubMed]

4. Radwan, A.; Amidon, G.L.; Langguth, P. Mechanistic investigation of food effect on disintegration and dissolution of BCS class III compound solid formulations: The importance of viscosity. Biopharm. Drug Dispos. 2012, 33, 403-416. [CrossRef] [PubMed]

5. Nickerson, B.; Kong, A.; Gerst, P.; Kao, S. Correlation of dissolution and disintegration results for an immediate-release tablet. J. Pharm. Biomed. Anal. 2018, 150, 333-340. [CrossRef] [PubMed]

6. Al-Gousous, J.; Amidon, G.L.; Langguth, P. Toward Biopredictive Dissolution for Enteric Coated Dosage Forms. Mol. Pharmaceutics. 2016, 13, 1927-1936. [CrossRef] [PubMed]

7. Al-Tabakha, M.M.; Arida, A.I.; Fahelelbom, K.M.S.; Sadek, B.; Jarad, R.A.A. Performances of New Generation of Delayed Release Capsules. J. Young Pharm. 2015, 7, 36-44. [CrossRef]

8. VassarStats. Available online: http://vassarstats.net/ (accessed on 1 December 2019).

9. Kamba, M.; Seta, Y.; Takeda, N.; Hamaura, T.; Kusai, A.; Nakane, H.; Nishimura, K. Measurement of agitation force in dissolution test and mechanical destructive force in disintegration test. Int. J. Pharm. 2003, 250, 99-109. [CrossRef]

10. D'Arcy, D.M.; Corrigan, O.I.; Healy, A.M. Evaluation of hydrodynamics in the basket dissolution apparatus using computational fluid dynamics-Dissolution rate implications. Eur. J. Pharm. Sci. 2006, 27, $259-267$. [CrossRef] [PubMed]

11. Bhagavan, H.N.; Wolkoff, B.I. Correlation between the disintegration time and the bioavailability of vitamin C tablets. Pharm. Res. 1993, 10, 239-242. [CrossRef] [PubMed]

12. Brouwers, J.; Tack, J.; Augustijns, P. Parallel monitoring of plasma and intraluminal drug concentrations in man after oral administration of fosamprenavir in the fasted and fed state. Pharm. Res. 2007, 24, 1862-1869. [CrossRef] [PubMed]

13. Brouwers, J.; Anneveld, B.; Goudappel, G.J.; Duchateau, G.; Annaert, P.; Augustijns, P.; Zeijdner, E. Food-dependent disintegration of immediate release fosamprenavir tablets: In vitro evaluation using magnetic resonance imaging and a dynamic gastrointestinal system. Eur. J. Pharm. Biopharm. 2011, 77, 313-319. [CrossRef] [PubMed] 